\title{
Motzkin Paths and Reduced Decompositions for Permutations with Forbidden Patterns
}

\author{
William Y. C. Chen ${ }^{1}$, Yu-Ping Deng ${ }^{2}$ and Laura L. M. Yang ${ }^{3}$ \\ Center for Combinatorics, LPMC \\ Nankai University, Tianjin 300071, P. R. China \\ ${ }^{1}$ chenstation@yahoo.com, ${ }^{2}$ dengyp@eyou.com, ${ }^{3}$ yanglm@hotmail.com
}

Submitted: Mar 22, 2003; Accepted: Jun 18, 2003; Published: Aug 3, 2003

MR Subject Classifications: 05A05, 05A15

\begin{abstract}
We obtain a characterization of $(321,3 \overline{1} 42)$-avoiding permutations in terms of their canonical reduced decompositions. This characterization is used to construct a bijection for a recent result that the number of (321,3142)-avoiding permutations of length $n$ equals the $n$-th Motzkin number, due to Gire, and further studied by Barcucci, Del Lungo, Pergola, Pinzani and Guibert. Similarly, we obtain a characterization of $(231,4 \overline{1} 32)$-avoiding permutations. For these two classes, we show that the number of descents of a permutation equals the number of up steps on the corresponding Motzkin path. Moreover, we find a relationship between the inversion number of a permutation and the area of the corresponding Motzkin path.
\end{abstract}

\section{Introduction}

Permutations with forbidden subsequences have been extensively studied over the last decade. Simion and Schmidt [21] and West [26] initiated the efforts towards forbidden subsequences of length 3. West [25] and Stankova [22] continued to study forbidden subsequences of length 4 , and discovered that the number of permutations in $S_{n+1}(3142,2413)$ equals the $n$-th Schröder number. Using the idea of generating trees, Kremer [17] discovered ten classes of permutations with forbidden patterns that are in one-to-one correspondence with Schröder paths. Dyck paths are also closely related to permutations with forbidden patterns, see Stanley [23], Krattenthaler [16] and West [26].

Motzkin paths come to the scene of permutations with forbidden patterns through the work of Gire [13], Barcucci, Del Lungo, Pergola and Pinzani [5, 6], Guibert [14] and Guibert, Pergola and Pinzani [15]. The classes $S_{n}(321,3 \overline{1} 42)$ and $S_{n}(231,4 \overline{1} 32)$ are enumerated by the $n$-th Motzkin number. Guibert [14] discovered that the involutions with forbidden patterns 3412, 4321, 1234 or 1243 are enumerated by Motzkin numbers. 
Moreover, Guibert, Pergola and Pinzani [15] proved that the number of avoiding-2143 involutions of length $n$ equals $n$-th Motzkin number by the method of generating trees.

The first result of this paper is an explicit bijection between $S_{n}(321,3 \overline{1} 42)$ and the set of Motzkin paths of length $n$. Using the canonical reduced decompositions, we obtain a characterization of permutations in $S_{n}(321,3 \overline{1} 42)$ in terms of their canonical reduced decompositions. Similarly, we give a bijection between $S_{n}(231,4 \overline{1} 32)$ and the set of Motzkin paths of length $n$ by their trapezoidal reduced decompositions.

Here are some known results in this direction: Bandlow and Killpatrick [3] give a bijection between $S_{n}(312)$ and Dyck paths by using reduced decompositions. Bandlow, Egge and Killpatrick [2], and Egge and Mansour [12] find bijections between permutations with forbidden patterns and Schröder paths.

For the two classes of permutations studied in this paper, we show that the number of descents of a permutation equals to the number of up steps on a Motzkin path, and we find a relationship between the inversion number of a permutation and the area of the corresponding Motzkin path.

\section{Reduced decompositions for $S_{n}(321,3 \overline{1} 42)$}

In this section, we give a characterization of permutations in $S_{n}(321,3 \overline{1} 42)$ in terms of their canonical reduced decompositions. We begin with some definitions and notations.

Let $S_{n}$ be the set of permutations on $[n]=\{1,2, \ldots, n\}$, where $n \geq 1$. For a permutation $\sigma$ of $k$ positive integers, the pattern or type of $\sigma$ is defined as a permutation $\tau$ on $[k]$ obtained from $\sigma$ by substituting the minimum element by 1 , the second minimum element by $2, \ldots$, and the maximum element by $k$. Sometimes we say that a permutation is order equivalent to its pattern. For example, the pattern of 68254 is 45132 . For a permutation $\tau \in S_{k}$ and a permutation $\pi \in S_{n}$, we say that $\pi$ is $\tau$-avoiding if there is no subsequence $\pi_{i_{1}} \pi_{i_{2}} \cdots \pi_{i_{k}}\left(i_{1}<i_{2}<\cdots<i_{k}\right)$ whose pattern is $\tau$. We write $S_{n}(\tau)$ for the set of $\tau$-avoiding permutations of $[n]$.

A barred permutation $\bar{\tau}$ of $[k]$ is a permutation of $S_{k}$ having a bar over one of its elements. Let $\tau$ be the permutation on $[k]$ obtained by unbarring $\bar{\tau}$, and $\hat{\tau}$ the pattern of the permutation obtained from $\tau$ by removing the barred element. A permutation $\pi \in S_{n}$ contains a subsequence $\omega$ of type $\bar{\tau}$ if and only if $\omega$ is of type $\hat{\tau}$ and it is not contained in any subsequence of type $\tau$. In other words, a subsequence $\omega$ of $\pi$ is of type $\bar{\tau}$ if it is of type $\hat{\tau}$ and it cannot be extended to a subsequence of type $\tau$. Equivalently, for a permutation $\pi \in S_{n}$, if every subsequence of type $\hat{\tau}$ can be extended to a subsequence of type $\tau$, then we say that $\pi$ avoids the barred pattern $\bar{\tau}$. We denote by $S_{n}(\bar{\tau})$ the set of permutations of $S_{n}$ avoiding the pattern $\bar{\tau}$.

For example, if $\pi=473591628, \bar{\tau}=235 \overline{1} 4$, then $\tau=23514, \hat{\tau}=1243$, all the subsequences of pattern 1243 are: 3596, 3598, 4596, 4598 and 4798; which are the subsequences of: $35916,35918,45916,45928$ and 47928 respectively, so we have $\pi \in S_{9}(235 \overline{1} 4)$.

We now review the notion of the canonical reduced decomposition of a permutation $[19,24]$ : 
Definition 2.1 For any $1 \leq i \leq n-1$, define the map $s_{i}: S_{n} \rightarrow S_{n}$, such that $s_{i}$ acts on a permutation by interchanging the elements in positions $i$ and $i+1$. We call $s_{i}$ a simple transposition, and write the action of $s_{i}$ on the right of the permutation, denoted by $\pi s_{i}$. Therefore one has $\pi\left(s_{i} s_{j}\right)=\left(\pi s_{i}\right) s_{j}$.

For example, $362514 s_{2}=326514$. The product of simple transpositions satisfies the Braid relations:

$$
\begin{aligned}
s_{i+1} s_{i} s_{i+1} & =s_{i} s_{i+1} s_{i}, \\
s_{i} s_{j} & =s_{j} s_{i}, \quad|i-j| \neq 1 .
\end{aligned}
$$

The permutation $12 \cdots n$ is called the minimum permutation whose canonical reduced decomposition is the identity. The permutation $w=n n-1 \cdots 1$ is called the maximum permutation which has the following canonical reduced decomposition:

$$
n n-1 \cdots 1=12 \cdots n\left(s_{1}\right)\left(s_{2} s_{1}\right)\left(s_{3} s_{2} s_{1}\right) \cdots\left(s_{n-1} s_{n-2} \cdots s_{1}\right) .
$$

In general, the canonical reduced decomposition of $\pi \in S_{n}$ has the following form:

$$
\pi=\left(\begin{array}{llll}
1 & 2 & \cdots & n
\end{array}\right) \sigma=\left(\begin{array}{llll}
1 & 2 & \cdots & n
\end{array}\right) \sigma_{1} \sigma_{2} \cdots \sigma_{k},
$$

where

$$
\begin{gathered}
\sigma_{i}=s_{h_{i}} s_{h_{i}-1} \cdots s_{t_{i}}, \quad h_{i} \geq t_{i} \quad(1 \leq i \leq k) \quad \text { and } \\
1 \leq h_{1}<h_{2}<\cdots<h_{k} \leq n-1 .
\end{gathered}
$$

Note that if one writes $\pi$ in the two row notation (as a permutation in the symmetric group), then from (2.1) one has the relation $\pi=\sigma_{1} \sigma_{2} \cdots \sigma_{k}$. We call $h_{i}$ the head and $t_{i}$ the tail of $\sigma_{i}$.

For example, for $\pi=315264 \in S_{6}$, the canonical reduced decomposition is $\left(s_{2} s_{1}\right)\left(s_{4} s_{3}\right)$ $\left(s_{5}\right)$. It is well-known that the above canonical reduced decomposition is unique. In fact, we have the following algorithm to generate the canonical reduced decomposition based on the recursive construction of a permutation on $[n]$ by inserting the element $n$ into a permutation on $[n-1]$. From this point of view, the idea of the canonical reduced decompositions falls into the general framework of the ECO methodology $[4,5]$.

Algorithm: Observe that the product $s_{j} s_{j-1} \cdots s_{i}$ is equivalent to the action of the cyclic permutation on the segment from position $i$ to position $j+1$. For the permutation 1, the reduced decomposition is the identity. Suppose that we have constructed the canonical reduced decomposition for the permutation $\pi \backslash n$, which is obtained from $\pi$ by deleting the element $n$. Assume that $n$ is in position $i$ in $\pi$. If $i=n$, the reduced decomposition of $\pi$ is the same as that of $\pi \backslash n$. For $i \neq n$, the action of $s_{n-1} s_{n-2} \cdots s_{i}$ would bring the element $n$ to the proper position and shift other relevant elements to the positions on their right. This gives the canonical reduced decomposition of $\pi$.

The canonical reduced decomposition has the following property $[3,19]$ : 
Lemma 2.2 If $\sigma$ is the canonical reduced decomposition of $\pi \in S_{n}$, then $\pi$ has $k$ inversions if and only if $\sigma$ has exactly $k$ simple transpositions.

The first result of this paper is the following characterization of canonical reduced decompositions of permutations in $S_{n}(321,3 \overline{1} 42)$.

Theorem 2.3 Let $\sigma=\sigma_{1} \cdots \sigma_{k}$ be the canonical reduced decomposition of $\pi \in S_{n}$, where $\sigma_{i}=\sigma_{h_{i}} \sigma_{h_{i}-1} \cdots \sigma_{t_{i}}$ for $1 \leq i \leq k$. Then

$$
\pi \in S_{n}(321,3 \overline{1} 42) \quad \text { if and only if } t_{i+1} \geq t_{i}+2 \text {, for } 1 \leq i \leq k-1 \text {. }
$$

Proof. The cases $k=0,1$ are obvious. We consider the case $k \geq 2$.

$\Longrightarrow)$ We use induction on $n$. Clearly, the statement is true for $n=1,2,3$. Suppose it is true for $n-1$. Assume that $n$ is in position $i$ in $\pi$. If $i=n$, then the assertion is automatically true because the canonical reduced decomposition of $\pi \backslash n$ is the same as that of $\pi$. When $i \neq n$, the canonical reduced decomposition of $\pi$ has one more factor $\sigma_{k}=s_{n-1} s_{n-2} \cdots s_{i}$. In other words, $h_{k}=n-1$ and $t_{k}=i$. We aim to show that $i \geq t_{k-1}+2$. Let

$$
\begin{aligned}
\pi \backslash n & =\beta_{1} \beta_{2} \cdots \beta_{i-1} \beta_{i} \cdots \beta_{n-1}, \\
\pi & =\beta_{1} \beta_{2} \cdots \beta_{i-1} n \beta_{i} \cdots \beta_{n-1} .
\end{aligned}
$$

By the inductive hypothesis, $\pi \backslash n \in S_{n-1}(321,3 \overline{1} 42)$. Assume that $t_{k-1}=i-1$. From the recursive constructions of the canonical reduced decompositions, one sees that whenever an element is brought to the proper position, it is always bigger than the element on its right. Thus one has $\beta_{i-1}>\beta_{i}$. It follows that $\beta_{i-1} n \beta_{i}$ has pattern 231 and there is no element between $\beta_{i-1}$ and $n$, which is a contradiction to the fact that $\pi$ avoids the pattern 3142. Again, we assume that $t_{k-1} \geq i$. For the same reason, one sees that $\beta_{t_{k-1}}>\beta_{t_{k-1}+1}$. Thus $n \beta_{t_{k-1}} \beta_{t_{k-1}+1}$ has pattern 321 , which is also a contradiction. Therefore, we conclude that $i \geq t_{k-1}+2$.

$\Longleftarrow)$ We use induction on $n$. Clearly, the statement is true for $n=1,2,3$. Assume that it is true for $n-1$. Suppose that $n$ is in position $i$ in $\pi$. When $i=n$, the canonical reduced decomposition of $\pi$ is the same as that of $\pi \backslash n$. Then we have $\pi \in S_{n}(321,3 \overline{1} 42)$ since $\pi \backslash n \in S_{n-1}(321,3 \overline{1} 42)$. When $i \neq n$, the canonical reduced decomposition of $\pi$ has one more factor $\sigma_{k}=s_{n-1} s_{n-2} \cdots s_{i}$. In other words, $h_{k}=n-1$ and $t_{k}=i$. Notice that we have the condition $i \geq t_{k-1}+2$. Let $h_{k-1}=n-m-1$ with $m \geq 1$, and let

$$
\pi \backslash n=\beta_{1} \beta_{2} \cdots \beta_{n-1}=(12 \cdots n-1) \sigma_{1} \sigma_{2} \cdots \sigma_{k-1} .
$$

Then we have

$$
\pi \backslash n=\beta_{1} \cdots \beta_{t_{k-1}-1}(n-m) \beta_{t_{k-1}+1} \cdots \beta_{n-m}(n-m+1) \cdots(n-1),
$$

where $\beta_{t_{k-1}}=n-m$. By the inductive hypothesis, $\pi \backslash n \in S_{n-1}(321,3 \overline{1} 42)$, thus the subsequence $\beta_{t_{k-1}+1} \cdots \beta_{n-m}$ is increasing. Since $i \geq t_{k-1}+2$, we have $n-m$ precedes $\beta_{i-1}$ in $\pi \backslash n$. Therefore, we obtain

$$
\pi=\beta_{1} \cdots(n-m) \cdots \beta_{i-1} n \beta_{i} \cdots \beta_{n-m} \cdots \beta_{n-1},
$$


where $n-m \geq \beta_{l}$ for $l=1, \cdots, n-m$. Assume that there exists a 321-pattern in $\pi$ which contains $n$, namely, a subsequence $n \beta_{j} \beta_{k}$ for some $i \leq j<k$. One sees that this may be possible only for $j<k \leq n-m$. Then $(n-m) \beta_{j} \beta_{k}$ would form a 321-pattern in $\pi \backslash n$, which leads to a contradiction. Now we assume that there exists a 231-pattern in $\pi$ that contains $n$, say $\beta_{j} n \beta_{k}$, then we have $j<i$ and $i \leq k \leq n-m$. Here are three cases:

1. $\beta_{j}$ precedes $n-m$ in $\pi$. Then $\beta_{j}(n-m) \beta_{k}$ is a 231-pattern in $\pi \backslash n$. Thus there exists an element $\beta_{l}$ between $\beta_{j}$ and $n-m$ such that $\beta_{j} \beta_{l}(n-m) \beta_{k}$ is a 3142-pattern. It follows that $\beta_{j} \beta_{l} n \beta_{k}$ is of type 3142 .

2. $\beta_{j}=n-m$, namely, $(n-m) n \beta_{k}$ is a 231-pattern. In this case, we have $\beta_{i-1}<\beta_{k}$ because $\pi \backslash n$ is 321 -avoiding. It follows that $(n-m) \beta_{i-1} n \beta_{k}$ is a 3142-pattern.

3. $\beta_{j}$ is between $n-m$ and $n$. Then $(n-m) \beta_{j} \beta_{k}$ forms a 321-pattern which is a contradiction to the inductive hypothesis.

In summary, we have shown that $\pi \in S_{n}(321,3 \overline{1} 42)$.

From Theorem 2.3, we establish a relationship between the set of descents and the set of tails of the canonical reduced decompositions for permutations in $S_{n}(321,3 \overline{1} 42)$. Recall that the set of descents of a permutation $\pi \in S_{n}$ is defined by $D(\pi)=\left\{i \mid \pi_{i}>\pi_{i+1}\right\}$.

Theorem 2.4 For any permutation $\pi \in S_{n}(321,3 \overline{1} 42)$, let $\sigma=\sigma_{1} \sigma_{2} \cdots \sigma_{k}$ be the canonical reduced decomposition of $\pi$, where $\sigma_{i}$ has head $h_{i}$ and tail $t_{i}$. Then the set of descents of $\pi$ is given by $\left\{t_{1}, t_{2}, \ldots, t_{k}\right\}$.

\section{The Strip Decomposition}

In this section, we present a bijection between $S_{n}(321,3 \overline{1} 42)$ and the set of Motzkin paths of length $n$ based on the characterization given in the preceding section. A Motzkin path of length $n$ is a path on the plane from the origin $(0,0)$ to $(n, 0)$ consisting of horizontal steps, up steps and down steps such that the path does not go across the $x$-axis. We will use $H, U$ and $D$ to represent the horizontal, up and down steps, respectively. The set of Motzkin paths of length $n$ is denoted by $M_{n}$, and the cardinality of $M_{n}$ is called the $n$-th Motzkin number. The first few Motzkin numbers are 1, 1, 2, 4, 9, 21, 51, 127, $\cdots$. For references on Motzkin paths, the reader is referred to $[1,7,8,9,10,11,18,20]$.

For example, $U H U D H D$ is a Motzkin path of length 6 . Note that we may also use the representation of a Motzkin path by the points on the path:

$$
(0,0)=A_{0} \rightarrow A_{1} \rightarrow A_{2} \rightarrow \cdots \rightarrow A_{n}=(n, 0)
$$

By Theorem 2.3, there exists a one-to-one correspondence between $S_{n}(321,3 \overline{1} 42)$ and the canonical reduced decompositions satisfying the following conditions

$$
t_{i+1} \geq t_{i}+2, \text { for } 1 \leq i \leq k-1 \text {. }
$$


Therefore, we are led to establish a bijection between Motzkin paths and the canonical reduced decompositions satisfying the conditions (3.1). What really matters is the set of parameters $\left\{\left(h_{i}, t_{i}\right) \mid 1 \leq i \leq k\right\}$ satisfying

$$
\begin{gathered}
1 \leq h_{1}<h_{2}<\cdots<h_{k} \leq n-1, \\
h_{i} \geq t_{i} \quad(1 \leq i \leq k), \\
t_{i} \geq t_{i-1}+2 \quad(2 \leq i \leq k) .
\end{gathered}
$$

Our bijection involves a labelling of the cells in the region of a Motzkin path. The region of a Motzkin path is meant to be the area surrounded by the path and the $x$-axis. Furthermore, the region of a Motzkin path is subdivided into cells which are either unit squares or triangles with unit bottom sides. A triangular cell either contains an up-step or a down step. We will not label triangular cells containing up-steps. The other types of cells, either square or triangular, have bottom sides, say with points $(i, j)$ and $(i+1, j)$, we will label these cells with $s_{i+j}$ or simply $i+j$. We call this labelling the $(x+y)$-labelling.

We now define the strip decomposition of a Motzkin path. Suppose $P_{n, k}$ is a Motzkin path of length $n$ that contains $k$ up steps. If $k=0$, then the strip decomposition of $P_{n, 0}$ is simply the empty set. For any $P_{n, k} \in M_{n}$, let $A \rightarrow B$ be the last up step and $E \rightarrow F$ the last down step on $P_{n, k}$. Then we define the strip of $P_{n, k}$ as the path from $B$ to $F$ along the path $P_{n, k}$. Now we move the points from $B$ to $E$ one layer lower, namely, subtract the $y$-coordinate by 1 , and denote the adjusted points by $B^{\prime}, \ldots, E^{\prime}$. We form a new Motzkin path by using the path $P_{n, k}$ up to the point $A$, then joining the point $A$ to $B^{\prime}$ and following the adjusted segment until we reach the point $E^{\prime}$, then continuing with the points on the $x$-axis to reach the destination $(n, 0)$. Denote this Motzkin path by $P_{n, k-1}$, which may end with some horizontal steps.

From the strip of $P_{n, k}$, we may define the value $h_{k}$ as the label of the cell containing the step $E \rightarrow F$. Clearly, we have $h_{k} \leq n-1$. The value $t_{k}$ is defined as the label of the cell containing the step starting from the point $B$.

Iterating the above procedure, we get a set of parameters $\left\{\left(h_{i}, t_{i}\right) \mid 1 \leq i \leq k\right\}$ satisfying the conditions (3.2). For each step in the above procedure, we obtain a product of transpositions $\sigma_{i}=s_{h_{i}} s_{h_{i}-1} \cdots s_{t_{i}}$. Finally we get the corresponding canonical reduced decomposition $\sigma=\sigma_{1} \sigma_{2} \cdots \sigma_{k}$ and the corresponding permutation $\pi=\left(\begin{array}{lll}1 & 2 \cdots n\end{array}\right) \sigma$.

For the Motzkin path in Figure 1,

$$
P_{17,5}=U H D H U U H H D H U U D D H H D .
$$

From the strip decomposition, we get the parameter set

$$
\{(2,1),(8,5),(12,7),(13,12),(16,14)\} \text {. }
$$

The canonical reduced decomposition is

$$
\left(s_{2} s_{1}\right)\left(s_{8} s_{7} s_{6} s_{5}\right)\left(s_{12} s_{11} s_{10} s_{9} s_{8} s_{7}\right)\left(s_{13} s_{12}\right)\left(s_{16} s_{15} s_{14}\right) \text {. }
$$




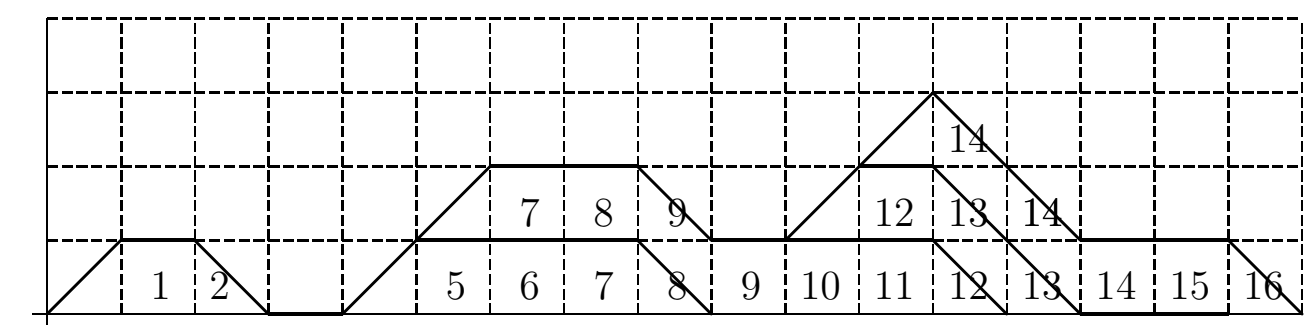

$(0,0)$

Figure 1: The $(x+y)$-labelling and strip decomposition.

The corresponding permutation is

$$
\begin{array}{lllllllllllllllll}
3 & 1 & 2 & 4 & 9 & 5 & 13 & 6 & 7 & 8 & 10 & 14 & 11 & 17 & 12 & 15 & 16 .
\end{array}
$$

Conversely, given a set of parameters $\left\{\left(h_{i}, t_{i}\right) \mid 1 \leq i \leq k\right\}$ satisfying the conditions (3.2), we may reverse the above procedure to construct a Motzkin path. Therefore we arrive at the following conclusion.

Theorem 3.1 There exists a bijection $\phi$ between $M_{n}$ and $S_{n}(321,3 \overline{1} 42)$.

For example, for the canonical reduced decomposition in (3.3), the construction of the Motzkin path is illustrated by Figure 2 .

By Lemma 2.2, we easily obtain the following theorem on the inversion number of a permutation in $S_{n}(321,3 \overline{1} 42)$. We define area of a Motzkin path as the area of the region bounded by the path and the $x$-axis. For a Motzkin path $P$, we use $\phi(P)$ to denote the permutation obtained from $P$ by the above bijection.

Theorem 3.2 The area of any Motzkin path $P$ minus the sum of y-coordinates of starting points in all the up steps is equal to the inversion number of the permutation $\phi(P) \in$ $S_{n}(321,3 \overline{1} 42)$.

Proof. By Lemma 2.2, the inversion number of a permutation equals the number of simple transpositions in its canonical reduced decomposition. Given any Motzkin path $P$, the canonical reduced decomposition of its corresponding permutation $\phi(P)$ is obtained from the labels of strips by omitting repeated labels in the same strip. However, the number of repeated labels is exactly the $y$-coordinate of the starting point in each strip. Moreover, the area of a Motzkin path equals the number of labelled cells. So the inversion number of the permutation $\phi(P) \in S_{n}(321,3 \overline{1} 42)$ equals the area of the Motzkin path $P$ minus the sum of $y$-coordinates of starting points of all the up steps.

\section{The Trapezoidal Decomposition}

It is discovered by Guibert [14] that $S_{n}(231,4 \overline{1} 32)$ is in one-to-one correspondence with the set of Motzkin paths of length $n$. The idea is to show that these two sets have the 

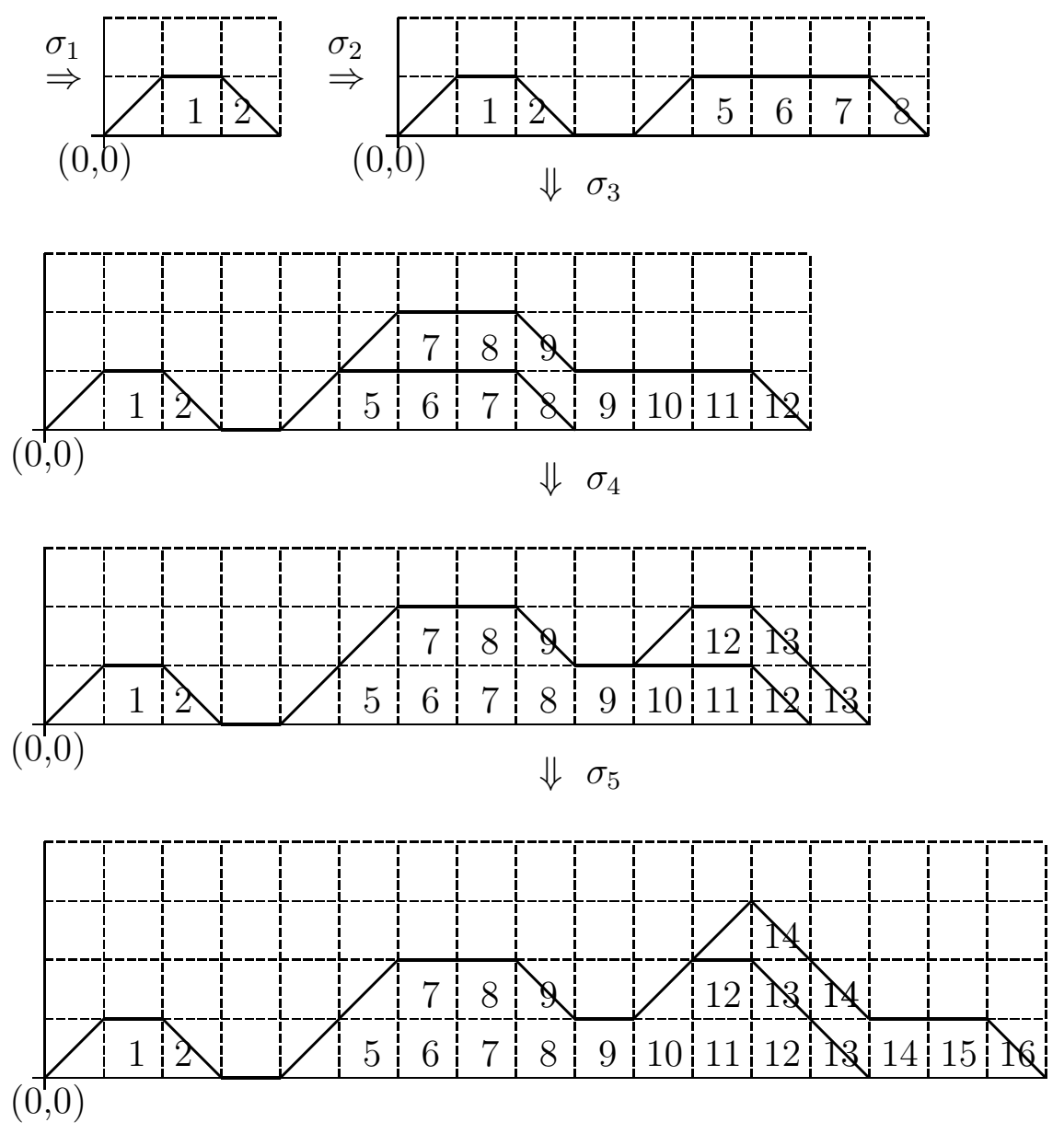

Figure 2: The Motzkin path from the canonical reduced decomposition.

same recursive structure. In this section, we provide a bijection based on the trapezoidal decomposition of Motzkin paths. Let us review some basic facts.

Proposition 4.1 For $n \geq 1$, the set $M_{n}$ of Motzkin paths $P$ of length $n$ can be recursively constructed as follows:

(i) $P=Q H$ for some Motzkin path $Q$ of length $n-1$.

(ii) $P=Q_{1} U Q_{2} D$, and we write $P=Q_{1} * Q_{2}$, for a Motzkin path $Q_{1}$ of length $i$ and a Motzkin path $Q_{2}$ of length $n-i-2$. Note that the step $U$ in the decomposition is the last up step that touches the $x$-axis.

Conversely, given any Motzkin path $P \in M_{n}$, one can uniquely decompose it into a shorter path by (i) or into a pair of shorter paths by (ii).

The recursive construction of $S_{n}(231,4 \overline{1} 32)$ can be described as follows. 
Proposition 4.2 For $n \geq 2$, the set of permutations $\theta$ in $S_{n}(231,4 \overline{1} 32)$ can be constructed by the following recursive procedure:

(i) $\theta=\pi n$ for some $\pi \in S_{n-1}(231,4 \overline{1} 32)$.

(ii) There exist $\pi_{1} \in S_{i}(231,4 \overline{1} 32)$ and $\pi_{2} \in S_{n-i-2}(231,4 \overline{1} 32)$ for some $0 \leq i \leq n-2$ such that

$$
\theta=\pi_{1} * \pi_{2}=\pi_{1} n(i+1) \tilde{\pi}_{2},
$$

where $\tilde{\pi}_{2}$ denotes the sequence obtained from $\pi_{2}$ by adding $i+1$ to every entry.

Conversely, given any permutation $\theta \in S_{n}(231,4 \overline{1} 32)$, one can uniquely decompose it into a shorter permutation by (i) or into a pair of shorter permutations by (ii).

The above recursive procedures lead to the following bijection of Guibert [14]:

Theorem 4.3 There is a bijection between $M_{n}$ and $S_{n}(231,4 \overline{1} 32)$.

The goal of this section is to present a bijection $\varphi$ between $M_{n}$ and $S_{n}(231,4 \overline{1} 32)$ in terms of reduced decompositions. A similar approach is used by Egge and Mansour [12] for permutations related to Schröder paths. For the strip decomposition for Motzkin paths in Section 3, the cutting point is the initial point of the last up step. In this section, we will deal with the decomposition for which the cutting point is the initial point of the last up step that touches the $x$-axis. We give a reduced decomposition based on the previous $(x+y)$-labelling and the trapezoidal decomposition of Motzkin paths.

For a Motzkin path $P$ containing at least one up step, we define the base trapezoid as the region surrounded by the $x$-axis, the last up step that touches the $x$-axis, then the necessary number of horizontal steps to reach the last down step, and finally the last down step. Note that the base trapezoid has at least one label. For example, the base trapezoid of the Motzkin path in Figure 3 has labels 6, 7, 8, 9, 10, 11, 12, 13.

- For $P=\emptyset$ or $P=H H \cdots H$, the trapezoidal decomposition of $P$ is the empty set.

- If $P$ has only one up step, then the decomposition is the base trapezoid.

- If $P$ has more than one up steps, then decompose it as $P=Q_{1} U Q_{2} D$, where $Q_{1}$ and $Q_{2}$ carry the labels in $P$. The trapezoidal decomposition of $P$ consists of the base trapezoid, followed by the trapezoidal decomposition of $Q_{2}$ and the trapezoidal decomposition of $Q_{1}$.

Next we associate a Motzkin path $P$ with a reduced decomposition. For each base trapezoid with labels $\{i, i+1, \ldots, j\}$ in the trapezoidal decomposition of $P$, we associate it with $s_{j} s_{j-1} \cdots s_{i}$. Suppose that $P$ has $k$ up steps and its trapezoidal decomposition is $T_{1}, T_{2}, \ldots, T_{k}$. Let $\sigma_{i}$ be the reduced decomposition of $T_{i}$. Then the reduced decomposition $\sigma=\sigma_{1} \sigma_{2} \cdots \sigma_{k}$ is called the trapezoidal reduced decomposition of $P$. Finally, the permutation obtained from $P$ during the above process is denoted by $\varphi(P)=(12 \cdots n) \sigma$. 
For example, in Figure 3 the Motzkin path $P$ is

$$
\text { UUDHDUUUDDHUDD. }
$$

The trapezoidal reduced decomposition is $\sigma=\sigma_{1} \sigma_{2} \cdots \sigma_{6}$, where $\sigma_{1}=s_{13} s_{12} s_{11} s_{10} s_{9} s_{8} s_{7} s_{6}$, $\sigma_{2}=s_{13}, \sigma_{3}=s_{10} s_{9} s_{8}, \sigma_{4}=s_{10}, \sigma_{5}=s_{4} s_{3} s_{2} s_{1}, \sigma_{6}=s_{3}$.

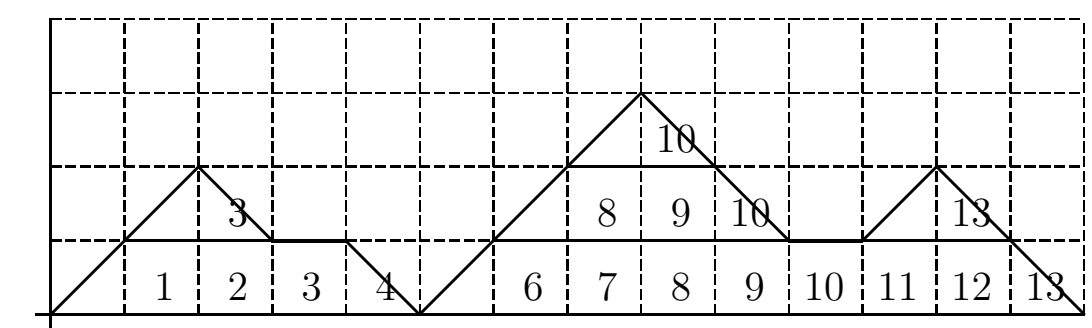

$(0,0)$

Figure 3: The trapezoidal reduced decomposition

By the construction of the trapezoidal reduced decompositions of Motzkin paths, we have the following assertion.

Theorem 4.4 Let $P$ be a Motzkin path of length $n$, and $\sigma=\sigma_{1} \sigma_{2} \ldots \sigma_{k}$ the trapezoidal reduced decomposition of $P$. Then we have $\varphi(P)=(12 \cdots n) \sigma \in S_{n}(231,4 \overline{1} 32)$.

Proof. We use induction on $n$. For $n=1$, it is clear that $\varphi(H)=1$. Assume that the statement is true for Motzkin paths of length less than $n$. If $P$ ends with a horizontal step, then there exists a unique Motzkin path $Q \in M_{n-1}$ such that $P=Q H$. Observing that $s_{n-1}$ does not appear in $\sigma_{1} \sigma_{2} \cdots \sigma_{k}$, we have

$$
\varphi(Q H)=\left(\begin{array}{llll}
1 & 2 \cdots n
\end{array}\right) \sigma(Q) n .
$$

By induction, $\varphi(Q) \in S_{n-1}(231,4 \overline{1} 32)$. It follows that $\pi=\varphi(Q) n \in S_{n}(231,4 \overline{1} 32)$.

If $P$ does not end with a horizontal step, we may have the decomposition $P=Q_{1} * Q_{2}=$ $Q_{1} U Q_{2} D$, where $Q_{1}$ is a Motzkin path of length $i$. From the construction of the trapezoidal reduced decomposition of $P$, we see that $\sigma_{1}=s_{n-1} \cdots s_{i+1}$. Let $\sigma_{2} \sigma_{3} \cdots \sigma_{k-j}$ denote the reduced decompositions corresponding to $Q_{2}$. In the factors $\sigma_{2}, \ldots, \sigma_{k-j}$, the indices of $s_{i}$ are bigger than $i+2$. Thus, $\sigma_{2}, \ldots, \sigma_{k-j}$ act only on the elements whose positions are bigger than $i+1$. Let $\sigma_{k-j+1} \sigma_{k-j+2} \cdots \sigma_{k}$ denote the reduced decompositions corresponding to $Q_{1}$, in which the indices of $s_{i}$ are smaller than $i$. Therefore, $\sigma_{k-j+1}, \ldots, \sigma_{k}$ act only on the first $i$ elements. It follows that

$$
\begin{aligned}
\varphi(P) & =(12 \cdots n) \sigma_{1} \sigma_{2} \cdots \sigma_{k} \\
& =(12 \cdots n)\left(s_{n-1} \cdots s_{i+1}\right)\left(\sigma_{2} \cdots \sigma_{k-j}\right)\left(\sigma_{k-j+1} \cdots \sigma_{k}\right) \\
& =(1 \cdots i n i+1 i+2 \cdots n-1)\left(\sigma_{2} \cdots \sigma_{k-j}\right)\left(\sigma_{k-j+1} \cdots \sigma_{k}\right) \\
& =\left(1 \cdots i n i+1 \widetilde{\varphi\left(Q_{2}\right)}\right)\left(\sigma_{k-j+1} \cdots \sigma_{k}\right)
\end{aligned}
$$




$$
\begin{aligned}
& =\left((1 \cdots i)\left(\sigma_{k-j+1} \cdots \sigma_{k}\right)\right) n i+1 \widetilde{\varphi\left(Q_{2}\right)} \\
& =\varphi\left(Q_{1}\right) n i+1 \widetilde{\varphi\left(Q_{2}\right)} \\
& =\varphi\left(Q_{1}\right) * \varphi\left(Q_{2}\right) .
\end{aligned}
$$

By Proposition 4.2, we have

$$
\pi=\varphi(P)=\varphi\left(Q_{1} * Q_{2}\right)=\varphi\left(Q_{1}\right) * \varphi\left(Q_{2}\right) \in S_{n}(231,4 \overline{1} 32) .
$$

This completes the proof.

For example, the Motzkin path in Figure 3 corresponds to the following permutation

$$
\begin{array}{llllllllllllll}
5 & 1 & 3 & 2 & 4 & 14 & 6 & 10 & 7 & 9 & 8 & 11 & 13 & 12 .
\end{array}
$$

By the above bijection, we obtain the following theorem on the inversion number of a permutation in $S_{n}(231,4 \overline{1} 32)$.

Theorem 4.5 The area of any Motzkin path $P$ is equal to the inversion number of the permutation $\varphi(P) \in S_{n}(231,4 \overline{1} 32)$.

By an inspection of the proof of Theorem 4.4, we obtain the following theorem on the set of descents of a permutation in $S_{n}(231,4 \overline{1} 32)$ in connection with the tails of the trapezoidal reduced decomposition of the corresponding Motzkin path.

Theorem 4.6 Let $\pi$ be a permutation in $S_{n}(231,4 \overline{1} 32)$, and $\sigma=\sigma_{1} \sigma_{2} \cdots \sigma_{k}$ the trapezoidal reduced decomposition of the corresponding Motzkin path. Let $t_{i}$ be the tail of $\sigma_{i}$. Then the set of descents of $\pi$ coincides with the set $\left\{t_{1}, t_{2}, \ldots, t_{k}\right\}$.

For example, the set of descents of the permutation in (4.2) is

$$
\{1,3,6,8,10,13\} \text {, }
$$

which coincides with the set of tails of the trapezoidal reduced decomposition in Figure 3.

\section{Acknowledgments.}

The authors would like to thank Alain Lasocux for valuable suggestions, and O. Guibert, J. West, C. Krattenthaler and J. Zeng for helpful comments. This work was done under the auspices of the National 973 Project on Mathematical Mechanization, the National Science Foundation, and the Ministry of Science and Technology of China. 


\section{References}

[1] M. Aigner, Motzkin numbers, Europ. J. Combin., 19 (1998), 663-675.

[2] J. Bandlow, Eric S. Egge and K. Killpatrick, A weight-preserving bijection between Schröder paths and Schröder permutations, Ann. Combin., 6 (2002), 235-248.

[3] J. Bandlow and K. Killpatrick, An area-to-inv bijection Dyck paths and 312-avoiding permutations, Elect. J. Combin., 8 (2001), \#R40.

[4] E. Barcucci, A. Del Lungo, E. Pergola and R. Pinzani, ECO: A methodology for enumeration of combinatorial objects, J. Diff. Equ. Appl., 5 (1999), 435-490.

[5] E. Barcucci, A. Del Lungo, E. Pergola and R. Pinzani, From Motzkin to Catalan permutations, Discrete Math., 217 (2000), 33-49.

[6] E. Barcucci, A. Del Lungo, E. Pergola and R. Pinzani, Some permutations with fobidden subsequences and their inversion number, Discrete Math., 234 (2001), 1-15.

[7] E. Barcucci, R. Pinzani and R. Sprugnoli, The Motzkin family, Pure Math. Appl. Ser. A, 2 (1991), 249-279.

[8] F. Bernhart, Catalan, Motzkin and Riordan numbers, Discrete Math., 204 (1999), 73-112.

[9] E. Deutsch and L. W. Shapiro, A bijection between ordered trees and 2-Motzkin paths and its many consequences, Discrete Math., 256 (2002), 655-670.

[10] R. Donaghey, Restricted plane tree representations of four Motzkin-Catalan equations, $J$. Combin. Theory Ser. B, 22 (1977), 114-121.

[11] R. Donaghey and L.W. Shapiro, Motzkin numbers, J. Combin. Theory Ser. A, 23 (1977), 291-301.

[12] E. S. Egge and T. Mansour, Permutations which avoid 1243 and 2143, continued fractions, and Chebyshev polynomials, http://www.arXiv:math.CO/0208046 v1, August 6, 2002.

[13] S. Gire, Arbres, permutations à motifs exclus et cartes planaires: quelques problèmes algorithmiques et combinatoires, Thèse de l'Université de Bordeaux I, 1993.

[14] O. Guibert, Combinatoire des permutations à motifs exclus en liaison avec mots, cartes planaires et tableaux de Young, Ph. D. Thesis, University Bordeaux I, France, 1995.

[15] O. Guibert, E. Pergola and R. Pinzani, Vexillary involutions are enumerated by Motzkin numbers, Ann. Combin., 5 (2001), 153-174.

[16] C. Krattenthaler, Permutations with restricted patterns and Dyck paths, Adv. Appli. Math., 27 (2001), 510-530.

[17] D. Kremer, Permutations with forbidden subsequences and a generalized Schröder number, Discrete Math., 218 (2000), 121-130.

[18] A. Kuznetsov, I. Pak and A. Postnikov, Trees associated with the Motzkin numbers, J. Combin. Theroy Ser. A, 76 (1996), 145-147. 
[19] A. Lascoux, Lecture Notes on the Symmetric Group, Nankai University, 2002.

[20] T. Motzkin, Relations between hypersurface cross ratios, and a combinatorial formula for partitions of a polygon, for permanent preponderance, and for nonassociative products, Bull. Amer. Math. Soc., 54 (1948), 352-360.

[21] R. Simion and W. Schmidt, Restricted permutations, Europ. J. Combin., 6 (1985), 383-406.

[22] Z. E. Stankova, Forbidden subsequences, Discrete Math., 132 (1994), 291-316.

[23] R. P. Stanley, Enumerative Combinatorics, Vol. 2, Cambridge University Press, Cambridge, 1999.

[24] R. P. Stanley, On the number of reduced decompositions of elements of Coxeter groups, Europ. J. Combin., 5 (1984), 359-372.

[25] J. West, Generating trees and the Catalan and Schröder numbers, Discrete Math., 146 (1995), 247-262.

[26] J. West, Permutations with forbidden subsequences and stack-sortable permutations, Ph.D. Thesis, M.I.T., Cambridge, MA, 1990. 